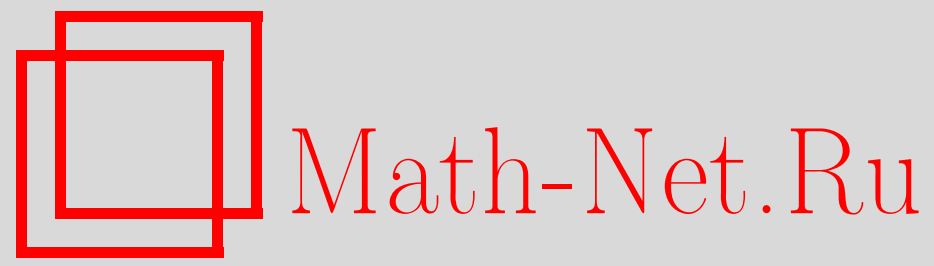

А. Г. Баскаков, И. А. Криштал, Н. Б. Ускова, Метод подобных операторов в исследовании спектральных свойств возмущенных дифференциальных операторов первого порядка, Итоги науки и техн. Сер. Соврем. мат. и ее прил. Tемат. обз., 2019, том 171, 3-18

DOI: https://doi.org/10.36535/0233-6723-2019-171-3-18

Использование Общероссийского математического портала Math-Net.Ru подразумевает, что вы прочитали и согласны с пользовательским соглашением

http://www.mathnet.ru/rus/agreement

Параметры загрузки:

IP: 34.239 .49 .27

26 апреля 2023 г., 11:33:59 


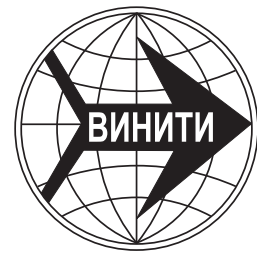

ИТОГИ НАУКИ И ТЕХНИКИ.

Современная математика и ее приложения.

Тематические обзоры.

Том 171 (2019). С. 3-18

DOI: $10.36535 / 0233-6723-2019-171-3-18$

УДК 517.9

\title{
МЕТОД ПОДОБНЫХ ОПЕРАТОРОВ \\ В ИССЛЕДОВАНИИ СПЕКТРАЛЬНЫХ СВОЙСТВ \\ ВОЗМУЩЕННЫХ ДИФФЕРЕНЦИАЛЬНЫХ ОПЕРАТОРОВ ПЕРВОГО ПОРЯДКА
}

\author{
(c) 2019 г. $\quad$ А. Г. БАСКАКОВ, И. А. КРИШТАЛ, Н. Б. УСКОВА
}

\begin{abstract}
АннотАция. В работе рассмотрены дифференциальные операторы первого порядка с периодическими краевыми условиями, действующие в гильбертовом пространстве суммируемых с квадратом на отрезке $[0, \omega]$ функций, возмущенные интегральными операторами Гильберта-Шмидта. Произведено преобразование подобия исходного оператора к оператору блочно-диагональной структуры; это позволяет изучить спектральные свойства возмущенного оператора. Методом исследования служит метод подобных операторов, основные положения которого и его систематизация также приведены в работе.
\end{abstract}

Ключевые слова: метод подобных операторов, спектр, интегро-дифференциальный оператор.

\section{METHOD OF SIMILAR OPERATORS IN THE STUDY OF SPECTRAL PROPERTIES OF PERTURBED FIRST-ORDER DIFFERENTIAL OPERATORS}

\author{
(c) 2019 A. G. BASKAKOV, I. A. KRISHTAL, N. B. USKOVA
}

\begin{abstract}
In this paper, we considers first-order differential operators with periodic boundary conditions acting in a Hilbert space of square summable functions on the segment $[0, \omega]$ perturbed by Hilbert-Schmidt integral operators. A similarity transformation of the original operator to the operator of the block-diagonal structure is performed; this allows one to study spectral properties of the perturbed operator. The research method is the method of similar operators, which also presented in this work.
\end{abstract}

Keywords and phrases: method of similar operators, spectrum, integro-differential operator.

AMS Subject Classification: 35L75, 35Q53, 37K10, 37K35

1. Введение. В настоящее время дифференциальные операторы первого порядка с инволюцией достаточно глубоко исследуются. Начало их систематическому изучению положили работы А. П. Хромова и М. Ш. Бурлуцкой (см. [14-20]), в которых резольвентным методом (с помощью контурного интегрирования) получены спектральные характеристики таких операторов с гладким потенциалом. В указанных работах рассматривались различные виды граничных условий и различные места нахождения инволюции: при производной или при потенциале. Полученные

Работа А. Г. Баскакова выполнена при поддержке Министерства образования и науки Российской Федерации (госзадание, проект № 1.3464.2017/4.6). Работа И. А. Криштала выполнена при поддержке Национального научного фонда США (National Science Foundation, проект DMS-1322127). Работа Н. Б. Усковой выполнена при поддержке Российского фонда фундаментальных исследований (проект № 19-01-00732). 
результаты легли в основу обоснования метода Фурье. Альтернативным методом исследования спектральных свойств различных возмущенных линейных замкнутых операторов, в том числе и операторов с инволюцией, является метод подобных операторов (см. $[2,4-7,9,10])$, позволяющий более гибко учитывать спектральные свойства оператора дифференцирования и специфику потенциала. При помощи указанного метода операторы с инволюцией и негладким потенциалом, в том числе матричным, исследовались в [11-13,24,25,27]. При этом необходимо было производить предварительное преобразование подобия метода подобных операторов (см. [11,24,25, 27]) из-за негладкости потенциала. После предварительного преобразования подобия исследование спектральных свойств исходного оператора сводится к исследованию спектральных свойств интегродифференциального оператора первого порядка (см. [11,24, 25, 27]). Данная статья посвящена изучению интегро-дифференциальных операторов первого порядка с помощью метода подобных операторов.

Также отличительной особенностью данной работы является систематизация основных теорем метода подобных операторов в изложении абстрактной схемы метода во разделе 2 и альтернативный способ доказательства одной из основных теорем метода подобных операторов (теоремы 2.2).

2. Метод подобных операторов. В этом разделе мы изложим основные положения метода подобных операторов. В отличие от обычно используемой схемы (см. [7, 9-11, 24, 25, 27]) ниже будет приведен более широкий обзор уравнений метода подобных операторов и условий их разрешимости.

Сначала введем необходимые далее операторные пространства. Пусть $\mathcal{H}$-абстрактное комплексное гильбертово пространство. Введем в рассмотрение линейный замкнутый оператор $A: D(A) \subset \mathcal{H} \rightarrow \mathcal{H}$, имеющий область определения $D(A)$, спектр $\sigma(A)$ и непустое резольвентное множество $\rho(A)$.

Определение 2.1 (см. [6]). Линейный оператор $B: D(B) \subset \mathcal{H} \rightarrow \mathcal{H}$ называется подчиненным оператору $A: D(A) \subset \mathcal{H} \rightarrow \mathcal{H}$, если $D(A) \subset D(B)$ и $\|B x\| \leqslant \operatorname{const}(\|x\|+\|A x\|), x \in D(A)$.

Обозначим пространство операторов, подчиненных оператору $A$, через $\mathfrak{L}_{A}(\mathcal{H})$. Введем в $\mathfrak{L}_{A}(\mathcal{H})$ норму, положив

$$
\|B\|_{A}=\inf \{C \geqslant 0:\|B x\| \leqslant C(\|x\|+\|A x\|) \text { для любого } x \in D(A)\} .
$$

Обычно без ограничения общности считается, что $D(B)=D(A)$.

Символом End $\mathcal{H}$ обозначим банахово пространство ( $C^{*}$-алгебру) всех ограниченных линейных операторов, действующих в $\mathcal{H}$, со стандартной нормой

$$
\|X\|=\sup _{\|x\| \leqslant 1}\|X x\|, \quad x \in \mathcal{H}, \quad X \in \operatorname{End} \mathcal{H} .
$$

Пространство End $\mathcal{H}$ вложено в $\mathfrak{L}_{A}(\mathcal{H})$, если область определения $D(A)$ оператора $A$ плотна в $\mathcal{H}$. Принадлежность оператора $X$ пространству $\mathfrak{L}_{A}(\mathcal{H})$ означает, что $X\left(A-\lambda_{0} I\right)^{-1} \in$ End $\mathcal{H}$ для любого $\lambda_{0} \in \rho(A)$. В пространстве $\mathfrak{L}_{A}(\mathcal{H})$ можно ввести эквивалентную норму, положив $\|X\|_{A}=$ $\left\|X\left(A-\lambda_{0} I\right)^{-1}\right\|$.

Начнем изложение метода подобных операторов с определения подобных операторов.

Определение 2.2 (см. [6]). Два линейных оператора $A_{i}: D\left(A_{i}\right) \subset \mathcal{H} \rightarrow \mathcal{H}, i=1,2$, называются подобными, если существует такой непрерывно обратимый оператор $U \in$ End $\mathcal{H}$, что $A_{1} U x=U A_{2} x, x \in D\left(A_{2}\right), U D\left(A_{2}\right)=D\left(A_{1}\right)$. Оператор $U$ называется оператором преобразования оператора $A_{1}$ в оператор $A_{2}$.

Подобные операторы обладают рядом совпадающих спектральных свойств, которые удобно сформулировать в виде следующей леммы.

Лемма 2.1 (см. [10]). Пусть $A_{i}: D\left(A_{i}\right) \subset \mathcal{H} \rightarrow \mathcal{H}, i=1,2$, - два подобных оператора и $U \in$ End $\mathcal{H}$ - оператор преобразования оператора $A_{1}$ в оператор $A_{2}$. Тогда справедливы следующие утверждения:

(1) $\sigma\left(A_{1}\right)=\sigma\left(A_{2}\right), \sigma_{d}\left(A_{1}\right)=\sigma_{d}\left(A_{2}\right), \sigma_{c}\left(A_{1}\right)=\sigma_{c}\left(A_{2}\right), \sigma_{r}\left(A_{1}\right)=\sigma_{r}\left(A_{2}\right)$, әде $\sigma\left(A_{i}\right)$ - cпектрь, $\sigma_{d}\left(A_{i}\right)$ - дискретные спектры, $\sigma_{c}\left(A_{i}\right)$ - непрерывные спектры, $\sigma_{r}\left(A_{i}\right)$-остаточные спектры операторов $A_{i}, i=1,2$, соответственно; 
(2) если а-собственный вектор оператора $A_{2}$, отвечающий собственному значению $\lambda_{0}$, то $b=U a-$ собственный вектор $A_{1}$, отвечающий тому же собственному значению $\lambda_{0} ;$

(3) если P - проектор Рисса, построенный по спектральному множеству $\sigma$ оператора $A_{2}$, mо спектральный проектор $\widetilde{P}$, построенный по этому же множеству $\sigma$ для оператора $A_{1}$, определяется формулой $\widetilde{P}=U P U^{-1}$;

(4) если оператор $A_{2}$ является генератором сильно непрерывной группы операторов $T_{2}: \mathbb{R} \rightarrow$ End $\mathcal{H}$ (класса $\left.C_{0}\right)$, то оператор $A_{1}$ является генератором сильно непрерывной группь операторов

$$
T_{1}(t)=U T_{2}(t) U^{-1}, \quad t \in \mathbb{R}, \quad T_{1}: \mathbb{R} \rightarrow \text { End } \mathcal{H} .
$$

Далее рассматривается оператор $A-B: D(A) \subset \mathcal{H} \rightarrow \mathcal{H}$ и $B \in \mathfrak{L}_{A}(\mathcal{H})$. Оператор $A$ будем называть невозмущенным, или свободным оператором. Спектральные свойства оператора $A$ считаются хорошо изученными. Оператор $B \in \mathfrak{L}_{A}(\mathcal{H})$ будем называть оператором-возмущением, а оператор $A-B$ - возмущенным оператором.

Основная идея метода подобных операторов состоит в следующем. Строится преобразование подобия возмущенного оператора $A-B$ в более просто устроенный оператор $A-\widetilde{B}$. При этом спектральные свойства оператора $A-\widetilde{B}$ должны быть близки к спектральным свойствам невозмущенного оператора $A$. Например, подпространства, построенные по непересекающимся частям спектра невозмущенного оператора $A$, остаются инвариантными и для оператора $A-\widetilde{B}$. Отметим, что прообразом метода подобных операторов является метод К. О. Фридрихса (см. [28]) для исследования возмущенных самосопряженных операторов с непрерывным спектром. Метод Фридрихса развивал Р. Тернер для нормальных компактных операторов (см. [29]), возмущенных ограниченными операторами, которые построены специальным образом по невозмущенному оператору. Метод подобных операторов также опирается на работы А. Пуанкаре, Н. М. Крылова, Н. Н. Боголюбова и окончательно оформляется в работах А. Г. Баскакова (см. $[2,4-7,9,10])$. Связь метода подобных операторов с методом усреднения (заменой Крылова-Боголюбова) см. в [1,3,8]; связь с методом Р. Тернера - в [23]. Отметим, что в настоящее время метод подобных операторов постоянно развивается и адаптируется к различным классам операторов. Поэтому изложения метода, например, в [6] и в [10] отличаются. Мы при изложении общих положений метода будем придерживаться работ $[9,10]$.

Следуя М. Г. Крейну, будем называть операторы, действующие в пространстве операторов, трансформаторами. Одним из трансформаторов, широко используемым в методе подобных операторов, является коммутатор

$$
\operatorname{ad}_{A}: D\left(\operatorname{ad}_{A}\right) \subset \text { End } \mathcal{H} \rightarrow \text { End } \mathcal{H}, \quad \operatorname{ad}_{A} X=A X-X A, \quad X \in D\left(\operatorname{ad}_{A}\right) .
$$

Область определения $D\left(\operatorname{ad}_{A}\right)$ состоит из всех таких $X \in \operatorname{End} \mathcal{H}$, что выполняются следующие два свойства:

(1) $X D(A) \subseteq D(A)$;

(2) оператор $\operatorname{ad}_{A} X: D(A) \subset \mathcal{H}$ допускает единственное расширение до ограниченного оператора $Y \in \operatorname{End} \mathcal{H}$; при этом полагается $\operatorname{ad}_{A} X=Y$.

Основным определением метода подобных операторов является определение допустимой тройки для невозмущенного оператора $A$.

Определение 2.3 (см. $[9,10])$. Пусть $\mathcal{M}$-линейное подпространство из $\mathfrak{L}_{A}(\mathcal{H})$ и $J: \mathcal{M} \rightarrow$ $\mathcal{M}, \Gamma: \mathcal{M} \rightarrow$ End $\mathcal{H}$ - трансформаторы. Тройку $(\mathcal{M}, J, \Gamma)$ назовем допустимой тройкой для оператора $A$, а $\mathcal{M}$-пространством допустимых возмущений, если выполнены следующие условия:

(1) $\mathcal{M}$ - банахово пространство со своей нормой $\|\cdot\|_{*}$, непрерывно вложенное в $\mathfrak{L}_{A}(\mathcal{H})$, т.е. существует такая постоянная $C>0$, что $\|X\|_{A} \leqslant C\|X\|_{*}$ для любого $X \in \mathcal{M}$;

(2) $J$ и $\Gamma$ - ограниченные линейные трансформаторы, причем $J$-идемпотент;

(3) $(\Gamma X) D(A) \subset D(A)$ и имеют место равенства

$$
\left(\operatorname{ad}_{A} \Gamma X\right) x=A \Gamma X x-\Gamma X A x=(X-J X) x, \quad x \in D(A), \quad X \in \mathcal{M},
$$

где $\Gamma X \in$ End $\mathcal{H}$ - единственное решение уравнения

$$
\operatorname{ad}_{A} Y=A Y-Y A=X-J X
$$


удовлетворяющее условию $J Y=0$;

(4) $X \Gamma Y,(\Gamma X) Y \in \mathcal{M}$ для любых $X, Y \in \mathcal{M}$ и существует такая постоянная $\gamma>0$, что

$$
\|\Gamma\| \leqslant \gamma, \quad \max \left\{\|X \Gamma Y\|_{*},\|(\Gamma X) Y\|_{*}\right\} \leqslant \gamma\|X\|_{*}\|Y\|_{*} ;
$$

(5) $J((Г X) J Y)=0$ для всех $X, Y \in \mathcal{M}$;

(6) для любых $X \in \mathcal{M}$ и $\varepsilon>0$ существует такое $\lambda_{\varepsilon} \in \rho(A)$, что

$$
\left\|X\left(A-\lambda_{\varepsilon} I\right)^{-1}\right\|<\varepsilon .
$$

Теперь зафиксируем некоторую допустимую для невозмущенного оператора $A$ тройку $(\mathcal{M}, J, \Gamma)$ и рассмотрим некоторое возмущение $B \in \mathcal{M}$.

Выпишем основные нелинейные уравнения, получаемые из условия подобия возмущенного оператора $A-B$ оператору $A-J X$, где $X$ - некоторый оператор из $\mathcal{M}$, подлежащий определению ( $X$ - решение одного из приводимых далее нелинейных операторных уравнений). Отметим, что в общем случае условие $J^{2}=J$ на выбор трансформатора $J$ не обязательно, однако оно существенно облегчает изучение операторов $A-J X$ и в данной работе мы предполагаем его выполненным.

Тип уравнения для неизвестного оператора $X \in \mathcal{M}$ существенно зависит от способа построения оператора преобразования $U \in \operatorname{End} \mathcal{H}$ оператора $A-B$ в оператор $A-J X$.

В методе подобных операторов обычно используют три варианта выбора оператора преобразования $U \in \operatorname{End} \mathcal{H}$ (см. [6]).

Вариант 1. Пусть $U=I+\Gamma X, X \in \mathcal{M}$. Тогда подобие операторов $A-B$ и $A-J X$ запишется в виде

$$
(A-B)(I+\Gamma X)=(I+\Gamma X)(A-J X),
$$

откуда немедленно следует равенство

$$
A-B+A \Gamma X-B \Gamma X=A+\Gamma X A-J X-(\Gamma X)(J X) .
$$

Учитывая свойство (3) определения 2.3, имеем

$$
X-B \Gamma X-B=-(\Gamma X) J X,
$$

откуда

$$
X=B \Gamma X-(\Gamma X) J X+B .
$$

Применим к обеим частям уравнения (2.5) оператор $J$; учитывая свойство (5) определения 2.3, получим уравнение для нахождения неизвестного оператора $X \in \mathcal{M}$ :

$$
X=B \Gamma X-(\Gamma X) J X-(\Gamma X) J(B \Gamma X)+B .
$$

Вариант 2. Пусть

$$
U=\left(I+\frac{1}{2} \Gamma X\right)\left(I-\frac{1}{2} \Gamma X\right)^{-1} .
$$

Для вывода соответствующего уравнения рассмотрим оператор

$$
\left(I-\frac{1}{2} \Gamma X\right)(A-B)\left(I+\frac{1}{2} \Gamma X\right)-\left(I+\frac{1}{2} \Gamma X\right)(A-J X)\left(I-\frac{1}{2} \Gamma X\right)
$$

и преобразуем его части:

$$
\begin{aligned}
&\left(I+\frac{1}{2} \Gamma X\right)(A-J X)\left(I-\frac{1}{2} \Gamma X\right)=A-J X-\frac{1}{2}(\Gamma X) A-\frac{1}{2}(\Gamma X) J X-\frac{1}{2} A \Gamma X+ \\
&+\frac{1}{2}(J X) \Gamma X+\frac{1}{4}(\Gamma X) J X(\Gamma X)-\frac{1}{4}(\Gamma X) A(\Gamma X), \\
&\left(I-\frac{1}{2} \Gamma X\right)(A-B)\left(I+\frac{1}{2} \Gamma X\right)=A-B-\frac{1}{2} \Gamma X-\frac{1}{2} J X+\frac{1}{2}(\Gamma X) B-\frac{1}{2} B \Gamma X- \\
&-\frac{1}{4}(\Gamma X) A(\Gamma X)+\frac{1}{4}(\Gamma X) B \Gamma X .
\end{aligned}
$$


Опять же, используя свойство (3) определения 2.3 , получаем, что имеет место равенство

$$
\left(I-\frac{1}{2} \Gamma X\right)(A-B)\left(I+\frac{1}{2} \Gamma X\right)=\left(I+\frac{1}{2} \Gamma X\right)(A-J X)\left(I-\frac{1}{2} \Gamma X\right),
$$

если оператор $X \in \mathcal{M}$ есть решение нелинейного операторного уравнения

$$
X=\frac{1}{2}\left(\operatorname{ad}_{B} \Gamma X-\operatorname{ad}_{J X} \Gamma X-\frac{1}{2} \Gamma X(B-J X) \Gamma X\right)+B .
$$

Определение 2.4. Линейный замкнутый оператор $A: D(A) \subset \mathcal{H} \rightarrow \mathcal{H}$ с $\overline{D(A)}=\mathcal{H}$ называется самосопряженным, если оператор $i A: D(A) \subset \mathcal{H} \rightarrow \mathcal{H}$ является генератором сильно непрерывной изометрической группы операторов $T(t), t \in \mathbb{R},\|T(t)\|=1$.

Отметим, что несмотря на еще бо́льшую громоздкость уравнения (2.8) по сравнению с уравнениями (2.5) и (2.6), оно обладает одним важным преимуществом. Если изначально $A$ и $B-$ самосопряженные операторы, то при рассмотрении уравнений (2.5) и (2.6) их решения не будут в общем случае самосопряженными; решение же уравнения (2.8) наследует важное свойство самосопряженности операторов $A$ и $B$.

Вариант 3. Пусть $U=\exp (\Gamma X)$. В этом случае подобие операторов $A-B$ и $A-J X$ запишется в виде

$$
(A-B) \exp (\Gamma X)=(\exp (\Gamma X))(A-J X) .
$$

Опустим в этом случае вывод нелинейного операторного уравнения для неизвестного оператора $X \in \mathcal{M}$ ввиду его громоздкости. Ограничимся самим уравнением (см. [6,7]):

$$
X=\sum_{n=1}^{\infty} \frac{1}{n !}\left[(-1)^{n} \operatorname{ad}_{\Gamma X / 2}^{n} B-\operatorname{ad}_{\Gamma X / 2}^{n} J X\right]-\sum_{n=1}^{\infty} \frac{1}{(2 n+1) !}\left[\operatorname{ad}_{\Gamma X / 2}^{2 n}(X-J X)\right]+B
$$

Преимущество рассмотрения третьего варианта состоит в том, что оператор преобразования $U=$ $\exp (\Gamma X)$ всегда обратим и $U^{-1}=\exp (-\Gamma X)$, в отличие от выражения обратного к $I+\Gamma X$, через ряд

$$
(I+\Gamma X)^{-1}=\sum_{i=0}^{\infty}(-1)^{i}(\Gamma X)^{i} .
$$

Каждое из уравнений $(2.6),(2.8),(2.10)$ можно записать в виде

$$
X=\Phi_{i}(X), \quad i=1,2,3,
$$

где $\Phi_{i}: \mathcal{M} \rightarrow \mathcal{M}, i=1,2,3$, - нелинейные трансформаторы, определяемые правыми частями соответствующих нелинейных операторных уравнений. Так, например,

$$
\Phi_{1}(X)=B \Gamma X-(\Gamma X) J B-(\Gamma X) J(B \Gamma X)+B, \quad X \in \mathcal{M} .
$$

Теорема 2.1 (см. [6, теорема 19.3]). Пусть $(\mathcal{M}, J, \Gamma)$ - допустимая для оператора A тройка, $B \in \mathcal{M} u$

$$
\gamma\|B\|_{*}\|J\|<\frac{1}{9} .
$$

Тогда любое из уравнений (2.6), (2.8), (2.10) разрешимо и имеет единственное решение $X_{i} \in \mathcal{M}$, $i=1,2,3$. Кроме того, операторы $A-B$ и $A-J X_{i}$ подобны, и при подстановке $X=X_{i}, i=1,2,3$, имеют место равенства (2.4), (2.7), (2.9), соответственно.

Отметим, что обычно не рассматривают сразу все три преобразования подобия и все три нелинейных операторных уравнения. Если же каждый вариант рассматривать отдельно, то для первого и второго случаев условие (2.12) можно ослабить. Приведем соответствующие теоремы. Кроме того, в стандартной схеме условие разрешимости нелинейных уравнений (2.11) устанавливалось с помощью метода мажорантных уравнений. В данной работе мы предложим другой способ доказательства известной базовой теоремы метода подобных операторов, отличный от изложенного в [6]. 
Теорема 2.2 (см. [6, теорема 19.2]). Пусть $(\mathcal{M}, J, \Gamma)$-допустимая для оператора $A$ тройка и $B$-некоторый оператор из $\mathcal{M}$. Если

$$
4 \gamma\|B\|_{*}\|J\|<1,
$$

то оператор $A-B$ подобен оператору $A-J X_{*}$, где $X_{*} \in \mathcal{M}$ является решением нелинейного уравнения

$$
X=B \Gamma X-(\Gamma X) J B-(\Gamma X) J(B \Gamma X)+B=\Phi_{1}(X) .
$$

Решение $X_{*}$ может быть найдено методом простых итераций, если положить $X_{0}=0$ и $X_{n}=$ $\Phi_{1}\left(X_{n-1}\right), n \in \mathbb{N}$. Преобразование подобия оператора $A-B$ в оператор $A-J X_{*}$ осуществляет обратимый оператор $I+\Gamma X_{*} \in$ End $\mathcal{H}$. Отображение $\Phi_{1}: \mathcal{M} \rightarrow \mathcal{M}$ является сжсимающим 8 wape $\mathcal{B}=\left\{X \in \mathcal{M}:\|X-B\|_{*} \leqslant 3\|B\|_{*}\right\}$.

Доказательство (см. такэне [27]). Мы используем теорему Банаха о неподвижной точке, чтобы доказать существование решения $X_{*} \in \mathcal{M}$ нелинейного операторного уравнения (2.6). Отметим, что

$$
\|X\|_{*}=\|X-B+B\|_{*} \leqslant\|X-B\|_{*}+\|B\|_{*} \leqslant 4\|B\|_{*} .
$$

Покажем, что отображение $\Phi_{1}$ переводит шар $\mathcal{B}$ в себя и

$$
\left\|\Phi_{1}(X)-\Phi_{1}(Y)\right\|_{*} \leqslant q\|X-Y\|_{*}
$$

для всех $X, Y \in \mathcal{B}$ и некоторого $q<1$. Действительно,

$$
\begin{aligned}
\left\|\Phi_{1}(X)-B\right\|_{*} \leqslant & \|B \Gamma X-(\Gamma X) J B-(\Gamma X) J(B \Gamma X)\|_{*} \leqslant \\
& \leqslant 4 \gamma\|B\|_{*}^{2}+4 \gamma\|J\|\|B\|_{*}^{2}+16 \gamma^{2}\|J\|\|B\|_{*}^{3} \leqslant\left(\|J\|^{-1}+1+\|J\|^{-1}\right)\|B\|_{*} \leqslant 3\|B\|_{*} .
\end{aligned}
$$

Введем обозначение $q=4 \gamma\|J\|\|B\|_{*}$. Очевидно, в силу (2.13), выполняется неравенство $q<1$. Имеем

$$
\begin{aligned}
& \left\|\Phi_{1}(X)-\Phi_{1}(Y)\right\|_{*} \leqslant \\
& \leqslant\|B \Gamma(X-Y)\|_{*}+\|\Gamma(X-Y) J B\|_{*}+\|(\Gamma X) J(B \Gamma X)-(\Gamma Y) J(B \Gamma Y) \pm(\Gamma X) J(B \Gamma Y)\|_{*} \leqslant \\
& \leqslant \gamma\|B\|_{*}\|X-Y\|_{*}+\gamma\|J\|\|B\|_{*}\|X-Y\|_{*}+\|(\Gamma X) J(B \Gamma(X-Y))\|_{*}+\|\Gamma(X-Y) J(B \Gamma Y)\|_{*} \leqslant \\
& \leqslant \gamma\|B\|_{*}\|X-Y\|_{*}+\gamma\|J\|\|B\|_{*}\|X-Y\|_{*}+\gamma^{2}\|B\|_{*}\left(\|X\|_{*}+\|Y\|_{*}\right)\|X-Y\|_{*} \leqslant \\
& \leqslant\left(\frac{q}{4}\|J\|^{-1}+\frac{q}{4}+\frac{q^{2}}{2}\|J\|^{-1}\right)\|X-Y\|_{*} \leqslant q\|X-Y\|_{*} .
\end{aligned}
$$

Таким образом, отображение $\Phi_{1}$ переводит шар $\mathcal{B}$ в себя и является в этом шаре сжимающим отображением с константой сжатия $q<1$. Поэтому отображение $\Phi_{1}$ имеет в шаре $\mathcal{B}$ единственную неподвижную точку $X_{*}, \Phi_{1}\left(X_{*}\right)=X_{*}$ и эта точка $X_{*}$ может быть найдена как предел последовательности $X_{n}, n \geqslant 0, X_{0}=0, X_{1}=B, \ldots, X_{n}=\Phi_{1}\left(X_{n-1}\right), \ldots$

Осталось показать, что оператор $I+\Gamma X_{*}$ непрерывно обратим и $\left(\Gamma X_{*}\right) D(A)=D(A)$. Пусть $X_{*}$ - решение уравнения (2.6). Тогда

$$
\left\|\Gamma X_{*}\right\| \leqslant \gamma\left\|X_{*}\right\|_{*} \leqslant 4 \gamma\|B\|_{*}<1
$$

и

$$
\left(I+\Gamma X_{*}\right)^{-1}=\sum_{n=0}^{\infty}(-1)^{n}\left(\Gamma X_{*}\right)^{n} .
$$

Так как $\left(I+\Gamma X_{*}\right) D(A) \subset D(A)$ согласно свойству (3) определения 2.3 , то осталось показать, что $\left(I+\Gamma X_{*}\right)^{-1} D(A) \subset D(A)$. Пусть $\lambda \in \rho(A)$. Тогда

$$
\begin{aligned}
& \left(\Gamma X_{*}\right)(A-\lambda I)^{-1}=(A-\lambda I)^{-1}\left((A-\lambda I)\left(\Gamma X_{*}\right)(A-\lambda I)^{-1}\right)= \\
= & (A-\lambda I)^{-1}\left(X_{*}-J X_{*}+\left(\Gamma X_{*}\right) A-\lambda \Gamma X_{*}\right)(A-\lambda I)^{-1}=(A-\lambda I)^{-1}\left(\left(X_{*}-J X_{*}\right)(A-\lambda I)^{-1}+\Gamma X_{*}\right) .
\end{aligned}
$$

Используя свойство (6) определения 2.3 и (2.14), выберем такое $\lambda \in \rho(A)$, что

$$
\left\|\left(X_{*}-J X_{*}\right)(A-\lambda I)^{-1}+\Gamma X_{*}\right\|<1 .
$$


Тогда

$$
\left(I+\Gamma X_{*}\right)^{-1}(A-\lambda I)^{-1}=(A-\lambda I)^{-1}\left(I+\left(X_{*}-J X_{*}\right)(A-\lambda I)^{-1}+\Gamma X_{*}\right)^{-1} .
$$

Следовательно, $\left(I+\Gamma X_{*}\right)^{-1} D(A) \subseteq D(A)$. Доказательство теоремы 2.2 закончено.

Аналогично можно показать, что имеет место следующее утверждение.

Теорема 2.3. Пусть $A$ и $B$-самосопряжсенные операторы, $(\mathcal{M}, J, \Gamma)$-допустимая для оператора А тройка, причем

$$
(J Y)^{*}=J\left(Y^{*}\right), \quad Y \in \mathcal{M} .
$$

Если выполнено условие

$$
\gamma\|B\|_{*}\|J\|<0,1568
$$

то уравнение (2.8) имеет единственное решение $X_{*} \in \mathcal{M}$, которое является самосопряљенным оператором. Это решение может быть найдено методом простых итераций. Операторы $A-B$ и $A-J X_{*}$ подобны и имеет место равенство (2.7).

3. Предварительные сведения. Пусть $\mathcal{H}=L_{2}[0, \omega]$ - гильбертово пространство (классов эквивалентности) комплекснозначных функций, измеримых по Лебегу на отрезке $[0, \omega]$ и суммируемых с квадратом модуля. Скалярное произведение в $L_{2}[0, \omega]$ и порождаемая им норма задаются формулами

$$
(x, y)=\frac{1}{\omega} \int_{0}^{\omega} x(s) \overline{y(s)} d s, \quad x, y \in \mathcal{H}, \quad\|x\|^{2}=\frac{1}{\omega} \int_{0}^{\omega}|x(s)|^{2} d s, \quad x \in \mathcal{H},
$$

Через $W_{2}^{1}[0, \omega]$ обозначено пространство Соболева абсолютно непрерывных функций из $\mathcal{H}$ с производными из $\mathcal{H}$ и скалярным произведением

$$
\langle x, y\rangle=(x, y)+\left(x^{\prime}, y^{\prime}\right), \quad x, y \in \mathcal{H}
$$

В пространстве $\mathcal{H}$ рассматривается интегро-дифференциальный оператор первого порядка

$$
(L y)(s)=y^{\prime}(s)-\int_{0}^{s} K(t, s) y(s) d s
$$

с областью определения $D(L)$, порождаемой периодическими краевыми условиями $D(L)=\{y \in$ $\left.W_{2}^{1}[0, \omega]: y(0)=y(\omega)\right\}$. Предполагается также, что ядро интегрального оператора удовлетворяет условию

$$
\int_{0}^{\omega} \int_{0}^{\omega}|K(t, s)|^{2} d s d t<\infty
$$

Представим оператор $L$ в виде $L=L_{0}-B$, где

$$
L_{0}: D\left(L_{0}\right)=D(L) \subset \mathcal{H} \rightarrow \mathcal{H}, \quad\left(L_{0} y\right)(t)=y^{\prime}(t), \quad(B y)(t)=\int_{0}^{\omega} K(t, s) y(s) d s .
$$

Невозмущенным, или свободным, считаем оператор $L_{0}$, а возмущением - оператор $B$.

Определение 3.1. [22]. Оператор $A: D(A) \subset \mathcal{H} \rightarrow \mathcal{H}$ с плотной областью определения $\overline{D(A)}$ называется нормальным, если $D(A)=D\left(A^{*}\right)$ и $\|A x\|=\left\|A^{*} x\right\|$ для всех $x \in D(A)$.

Отметим, что в $[5,6]$ использовалось другое, эквивалентное, определение нормального оператора.

Определение 3.2. Линейный замкнутый оператор $A: D(A) \subset \mathcal{H} \rightarrow \mathcal{H}$ с $\overline{D(A)}=\mathcal{H}$ называется нормальным, если он представим в виде $A=A_{1}+i A_{2}$, где $A_{i}: D\left(A_{i}\right)=D(A) \subset \mathcal{H} \rightarrow \mathcal{H}$, $i=1,2,-$ самосопряженные операторы. 
Невозмущенный оператор $L_{0}$ является нормальным оператором с компактной резольвентой. Его простыми изолированными собственными значениями являются числа $\lambda_{n}=i 2 \pi n / \omega, n \in \mathbb{Z}$, а соответствующими собственными векторами - функции $e_{n}(t)=e^{\lambda_{n} t}=e^{i 2 \pi n t / \omega}, n \in \mathbb{Z}$. Спектральные проекторы $P_{n}=P\left(\left\{\lambda_{n}\right\}, L_{0}\right), n \in \mathbb{Z}$, задаются формулой

$$
P_{n} x=\left(x, e_{n}\right) e_{n}, \quad n \in \mathbb{Z} .
$$

Введем в рассмотрение двусторонний идеал операторов Гильберта-Шмидта $\mathfrak{S}_{2}(\mathcal{H})$ из алгебры End $\mathcal{H}$. Через $\|X\|_{2}, X \in \mathfrak{S}_{2}(\mathcal{H})$, обозначим норму Гильберта-Шмидта: $\|X\|_{2}^{2}=\operatorname{tr}\left(X X^{*}\right)$ след оператора $X X^{*}$, принадлежащего двустороннему идеалу $\mathfrak{S}_{1}(\mathcal{H})$ ядерных операторов из End $\mathcal{H}$ (см. [21]) с нормой $\|X\|_{1}=\sum_{n \in \mathbb{Z}} s_{n}$, где $\left(s_{n}\right)$ - последовательность $s$-чисел оператора $X \in \mathfrak{S}_{1}(\mathcal{H})$. Пространство $\mathfrak{S}_{2}(\mathcal{H})$ является гильбертовым, и формула $(X, Y)=\operatorname{tr}\left(X Y^{*}\right)$ задает скалярное произведение в $\mathfrak{S}_{2}(\mathcal{H})$. Свойства операторных идеалов $\mathfrak{S}_{2}(\mathcal{H})$ и $\mathfrak{S}_{1}(\mathcal{H})$, в частности, используемые далее, можно найти в [21].

Каждому оператору $X \in$ End $\mathcal{H}$ поставим в соответствие его операторную матрицу $X=\left(X_{i j}\right)$, $i, j \in \mathbb{Z}$, где $X_{i j}=P_{i} X P_{j}$ и проекторы $P_{i}, i \in \mathbb{Z}$, определены формулой (3.3).

Отметим, что система проекторов $\left(P_{i}, i \in \mathbb{Z}\right)$ является дизъюнктной системой ортопроекторов, и в $\mathfrak{S}_{2}(\mathcal{H})$ можно ввести эквивалентную норму

$$
\|X\|_{2}^{2}=\sum_{i, j \in \mathbb{Z}}\left\|X_{i j}\right\|_{2}^{2}
$$

Заметим, что условие $(3.2)$ на оператор-возмущение $B$ означает, что $B \in \mathfrak{S}_{2}(\mathcal{H})$ и

$$
\|B\|_{2}^{2}=\int_{0}^{\omega} \int_{0}^{\omega}|K(t, s)|^{2} d s d t .
$$

Пусть ядро $K(t, s)$ имеет ряд Фурье вида

$$
K(t, s)=\sum_{l, p \in \mathbb{Z}} \widehat{k}(l, p) e^{i \frac{2 \pi l}{\omega} t} e^{i \frac{2 \pi p}{\omega} s} .
$$

Тогда из условия (3.2) следует, что

$$
\sum_{l, p \in \mathbb{Z}}|\widehat{k}(l, p)|^{2}<\infty
$$

Кроме того, в базисе из собственных векторов $e_{i}, i \in \mathbb{Z}$, невозмущенного оператора $L_{0}$ матрица оператора-возмущения $B$ состоит из элементов

$$
b_{l p}=\frac{1}{\omega} \widehat{k}(-l, p), \quad l, p \in \mathbb{Z} .
$$

В следующем разделе мы перейдем к построению допустимых троек для невозмущенного оператора $L_{0}$. Подчеркнем, что метод подобных операторов легок в применении, если собственные значения невозмущенного оператора разбегаются. В данном же случае этого не происходит, и $\operatorname{dist}\left(\left\{\lambda_{n}\right\}, \sigma\left(L_{0}\right) \backslash\left\{\lambda_{n}\right\}\right)=2 \pi / \omega$ для всех $n \in \mathbb{Z}$. Поэтому приходится строить две допустимые тройки: первая с допустимым пространством возмущений $\mathfrak{S}_{2}(\mathcal{H})$, а вторая - с допустимым пространством возмущений $\mathcal{M}_{B} \subset \mathfrak{S}_{2}(\mathcal{H})$, построенным по возмущению $B \in \mathfrak{S}_{2}(\mathcal{H})$ с использованием последовательности $\left(\alpha_{n}, n \in \mathbb{Z}\right)$, которая учитывает скорость убывания элементов $P_{i} B P_{j}, i, j \in \mathbb{Z}$, $|i|,|j| \rightarrow \infty$.

4. Построение семейства допустимых троек $\left(\mathfrak{S}_{2}(\mathcal{H}), J_{k}, \Gamma_{k}\right)$. Пусть $\mathcal{M}=\mathfrak{S}_{2}(\mathcal{H})$. Отметим, что невозмущенный оператор $L_{0}$ необратим, но вместо $L_{0}$ можно рассматривать оператор $L_{0}-\mu I$, $\mu \in \rho\left(L_{0}\right)$. Допустимые тройки для $L_{0}$ и $L_{0}-\mu I$ будут одинаковые.

Очевидно, что $\mathfrak{S}_{2}(\mathcal{H}) \subset \mathfrak{L}_{L_{0}}(\mathcal{H})$, и свойство $(1)$ определения 2.3 имеет место. 
Перейдем к построению трансформаторов $J, \Gamma \in \operatorname{End}\left(\mathfrak{S}_{2}(\mathcal{H})\right)$. Для $X \in \mathfrak{S}_{2}(\mathcal{H})$ определим трансформаторы $J X$ и $\Gamma X$ матрицами

$$
(J X)_{i j}=\left\{\begin{array}{lc}
X_{i j}, & i=j, \\
0, & i \neq j,
\end{array} \quad(\Gamma X)_{i j}= \begin{cases}X_{i j} /\left(\lambda_{i}-\lambda_{j}\right), & i \neq j, \\
0, & i=j .\end{cases}\right.
$$

Очевидно, что $J X=\sum_{n \in \mathbb{Z}} P_{n} X P_{n}$, и выписанный ряд безусловно сходится в $\mathfrak{S}_{2}(\mathcal{H})$,

$$
\|J X\|_{2}^{2}=\sum_{i \in \mathbb{Z}}\left\|P_{i} X P_{i}\right\|_{2}^{2} \leqslant\|X\|_{2}^{2}
$$

т.е. из $X \in \mathfrak{S}_{2}(\mathcal{H})$ следует, что $J X \in \mathfrak{S}_{2}(\mathcal{H})$.

Покажем, что $\Gamma X \in \mathfrak{S}_{2}(\mathcal{H})$ для $X \in \mathfrak{S}_{2}(\mathcal{H})$. Действительно,

$$
\|\Gamma X\|_{2}^{2}=\sum_{\substack{i, j \in \mathbb{Z}, i \neq j}} \frac{\left\|X_{i j}\right\|_{2}^{2}}{\left|\lambda_{i}-\lambda_{j}\right|^{2}} \leqslant\left(\frac{\omega}{2 \pi}\right)^{2} \sum_{\substack{i, j \in \mathbb{Z}, i \neq j}}\left\|X_{i j}\right\|_{2}^{2} \leqslant\left(\frac{\omega}{2 \pi}\right)^{2}\|X\|_{2}^{2} .
$$

Теорема 4.1. Тройка $\left(\mathfrak{S}_{2}(\mathcal{H}), J, \Gamma\right)$ является допустимой тройкой для оператора $L_{0}$.

Доказательство. Непрерывность трансформаторов $J, \Gamma \in \operatorname{End}\left(\mathfrak{S}_{2}(\mathcal{H})\right)$ следует из их построения. Норма $\|J\|$ трансформатора $J \in \operatorname{End}\left(\mathfrak{S}_{2}(\mathcal{H})\right)$ равна единице, так как, с одной стороны, $\|J X\|_{2}^{2} \leqslant$ $\|X\|_{2}^{2}$, а с другой стороны, если $X=\sum_{i \in \mathbb{Z}} P_{i} X P_{i}$, то $\|J X\|_{2}^{2}=\|X\|_{2}^{2}$.

Покажем, что $J^{2}=J$ :

$$
J(J X)=\sum_{j \in \mathbb{Z}} P_{j}\left(\sum_{i \in \mathbb{Z}} P_{i} X P_{i}\right) P_{j}=\sum_{j \in \mathbb{Z}} P_{j} X P_{j}=J X .
$$

Проверим свойства определения 2.3 , относящиеся к трансформатору $\Gamma \in \operatorname{End}\left(\mathfrak{S}_{2}(\mathcal{H})\right)$. Для проверки свойства (3) вычислим матрицы операторов $L_{0} \Gamma X, \Gamma X L_{0}, X-J X$. Очевидно, что

$$
\left(L_{0} \Gamma X\right)_{i j}=\frac{\lambda_{i} X_{i j}}{\lambda_{i}-\lambda_{j}}, \quad\left(\Gamma X L_{0}\right)_{i j}=\frac{\lambda_{j} X_{i j}}{\lambda_{i}-\lambda_{j}}, \quad(X-J X)_{i j}=X_{i j}, \quad i \neq j .
$$

При $i=j$ все три элемента равны нулю. Тогда

$$
\frac{\lambda_{i} X_{i j}}{\lambda_{i}-\lambda_{j}}-\frac{\lambda_{j} X_{i j}}{\lambda_{i}-\lambda_{j}}=X_{i j}, \quad i \neq j
$$

Таким образом, условие (2.1) выполнено.

Из формулы (4.2) следует, что $\|\Gamma\|$ оценивается величиной $\omega /(2 \pi)$. Операторы $X \Gamma Y,(\Gamma X) Y$ для $X, Y \in \mathfrak{S}_{2}(\mathcal{H})$ принадлежат $\mathfrak{S}_{1}(\mathcal{H}) \subset \mathfrak{S}_{2}(\mathcal{H})$ и $\|X \Gamma Y\|_{2} \leqslant(\omega /(2 \pi))\|X\|_{2}\|Y\|_{2} ;\|(\Gamma X) Y\|_{2}$ оценивается той же величиной. Свойство (4) выполняется.

Пусть $\lambda_{0} \in \rho\left(L_{0}\right), x \in D\left(L_{0}\right)$. Тогда $x=\left(L_{0}-\lambda_{0} I\right)^{-1} y, y \in \mathcal{H}$,

$$
\begin{aligned}
& \Gamma X\left(L_{0}-\lambda_{0} I\right)^{-1} y=\sum_{\substack{n, m \in \mathbb{Z}, n \neq m}} \frac{X_{m n}}{\left(\lambda_{m}-\lambda_{n}\right)\left(\lambda_{n}-\lambda_{0}\right)} y= \\
&=\sum_{\substack{n, m \in \mathbb{Z}, n \neq m}} \frac{X_{m n}}{\left(\lambda_{m}-\lambda_{n}\right)\left(\lambda_{m}-\lambda_{0}\right)} y+\sum_{\substack{n, m \in \mathbb{Z}, n \neq m}} \frac{X_{m n}}{\left(\lambda_{m}-\lambda_{0}\right)\left(\lambda_{n}-\lambda_{0}\right)} y= \\
&=\left(L_{0}-\lambda_{0} I\right)^{-1}(\Gamma X) y+\left(L_{0}-\lambda_{0} I\right)^{-1}(X-J X) x=\left(L_{0}-\lambda_{0} I\right)^{-1}(\Gamma X y+(X-J X) x) .
\end{aligned}
$$

Таким образом, $(\Gamma X) D\left(L_{0}\right) \subset D\left(L_{0}\right)$.

Переходим к свойству (5). Пусть $Z=(\Gamma X)(J Y), X, Y \in \mathfrak{S}_{2}(\mathcal{H})$. Тогда

$$
Z_{i j}=\sum_{p \in \mathbb{Z}}(\Gamma X)_{i p}(J X)_{p j}= \begin{cases}X_{i j} X_{j j} /\left(\lambda_{i}-\lambda_{j}\right), & i \neq j \\ 0, & i=j\end{cases}
$$


поэтому $J(\Gamma X(J Y))=0$.

Рассмотрим последнее, шестое свойство определения 2.3. Пусть $\lambda_{\varepsilon} \in \rho\left(L_{0}\right), X \in \mathfrak{S}_{2}(\mathcal{H})$. Оператор $X\left(L_{0}-\lambda_{\varepsilon} I\right) \in \mathfrak{S}_{2}(\mathcal{H})$ имеет операторную матрицу, состоящую из элементов $X_{i j} /\left(\lambda_{i}-\lambda_{\varepsilon}\right)$, $i, j \in \mathbb{Z}$, поэтому

$$
\left\|X\left(L_{0}-\lambda_{\varepsilon} I\right)^{-1}\right\|_{2}^{2} \leqslant \frac{1}{\max _{j \in \mathbb{Z}}\left(\lambda_{i}-\lambda_{j}\right)}\|X\|_{2}^{2} .
$$

Последнюю величину можно сделать сколь угодно малой за счет подходящего выбора числа $\lambda_{\varepsilon} \in \rho\left(L_{0}\right)$. Теорема 4.1 доказана.

Положим

$$
P_{(k)}=\sum_{\substack{|i| \leqslant k, i \in \mathbb{Z}}} P_{i}, \quad k \in \mathbb{Z}_{+} .
$$

Наряду с трансформаторами $J, \Gamma \in \operatorname{End}\left(\mathfrak{S}_{2}(\mathcal{H})\right)$ введем в рассмотрение семейства операторов $J_{k}, \Gamma_{k} \in \operatorname{End}\left(\mathfrak{S}_{2}(\mathcal{H})\right), k \in \mathbb{Z}_{+}$, определенных формулами

$$
\begin{array}{ll}
J_{k} X=P_{(k)} X P_{(k)}+\sum_{\substack{|i|>k, i \in \mathbb{Z}}} P_{i} X P_{i}, & X \in \mathfrak{S}_{2}(\mathcal{H}), \\
\Gamma_{k} X=\Gamma X-P_{(k)}(\Gamma X) P_{(k)}=\Gamma\left(X-J_{k} X\right), \quad X \in \mathfrak{S}_{2}(\mathcal{H}),
\end{array}
$$

при этом $J_{0} X=J X, \Gamma_{0} X=\Gamma X$. Операторы $J_{k} X$ и $\Gamma_{k} X, k \in \mathbb{Z}_{+}$, определены корректно, все указанные ряды сходятся в $\mathfrak{S}_{2}(\mathcal{H})$. Отметим, что операторы $J_{k} X$ и $\Gamma_{k} X$ отличаются от операторов $J X$ и $\Gamma X$ на оператор конечного ранга $2 k+1$. Аналогично предыдущему, имеет место следующая теорема.

Теорема 4.2. Тройка $\left(\mathfrak{S}_{2}(\mathcal{H}), \Gamma_{k} X, J_{k} X\right)$ является допустимой для оператора $L_{0}$ тройкой при любом $k \in \mathbb{Z}_{+}$.

5. Построение семейства допустимых троек $\left(\mathcal{M}_{B}, J_{k}, \Gamma_{k}\right)$. Ниже будем использовать пространства допустимых возмущений $\mathcal{M}_{B}$, впервые введенные в [9]. Основные результаты в $[11-13,24,25,27]$ также получены с использованием этих пространств. Чтобы описать их, для любого ненулевого оператора $X \in \mathfrak{S}_{2}(\mathcal{H})$ введем в рассмотрение двустороннюю последовательность вещественных чисел вида

$$
\alpha_{n}(X)=\|X\|_{2}^{-1 / 2} \max \left\{\left(\sum_{\substack{|k| \geqslant n, k \in \mathbb{Z}}}\left\|P_{k} X\right\|_{2}^{2}\right)^{1 / 4},\left(\sum_{\substack{|k| \geqslant n, k \in \mathbb{Z}}}\left\|X P_{k}\right\|_{2}^{2}\right)^{1 / 4}\right\}, \quad n \in \mathbb{Z} .
$$

Последовательность $\left(\alpha_{n}(X)\right), n \in \mathbb{Z}$, обладает следующими свойствами:

(1) $\alpha_{n}(X)=\alpha_{-n}(X), n \in \mathbb{Z}$

(2) $\lim _{|n| \rightarrow \infty} \alpha_{n}(X)=0, n \in \mathbb{Z}$;

(3) $\alpha_{n}(X) \leqslant 1$ для всех $n \in \mathbb{Z}$;

(4) $\alpha_{n}(X) \geqslant \alpha_{n+1}(X), n \geqslant 0$;

(5) $\alpha_{n}(X) \neq 0$ для всех $n \in \mathbb{Z}$, если $P_{(m)} X P_{(m)} \neq X$ для всех $m \in \mathbb{Z}_{+}$;

(6) конечна величина

$$
\sum_{n \in \mathbb{Z}} \frac{\left\|X P_{n}\right\|_{2}^{2}+\left\|P_{n} X\right\|_{2}^{2}}{\left(\alpha_{n}(X)\right)^{2}} .
$$

Без ограничения общности можно в дальнейшем считать, что $P_{(n)} B P_{(n)} \neq 0$ для всех $n \in$ $\mathbb{Z}_{+}$; в противном случае оператор $L_{0}-B$ есть ортогональная сумма оператора конечного ранга $\left.\left(L_{0}-B\right)\right|_{\mathcal{H}_{(n)}}$ для некоторого $n \geqslant 0$ и оператора $\left.L_{0}\right|_{\mathcal{H}^{(n)}}$, где $\mathcal{H}_{(n)}=P_{(n)} \mathcal{H}$ и $\mathcal{H}^{(n)}=\left(I-P_{(n)}\right) \mathcal{H}$.

Для любого оператора $X \in \mathfrak{S}_{2}(\mathcal{H})$ рассмотрим самосопряженный компактный оператор $F_{X}$, определенный формулой

$$
F_{X}=\sum_{n \in \mathbb{Z}} \alpha_{n}(X) P_{n}
$$


Отметим, что $F_{X} \in$ End $\mathcal{H}$ есть функция от нормального оператора $L_{0}$ вида $F_{X}=f_{X}\left(L_{0}\right)$, где $f_{X}: \sigma\left(L_{0}\right) \rightarrow \mathbb{R}_{+}, f_{X}\left(\lambda_{n}\right)=\alpha_{n}(X), n \in \mathbb{Z}$, и $\left\|F_{X}\right\|=\max _{n \in \mathbb{Z}}\left|\alpha_{n}(X)\right|=1$.

Для упрощения дальнейшей записи оператор $F_{B}$ обозначим через $F$.

Введем множество операторов $\mathcal{M}_{B}$ из $\mathfrak{S}_{2}(\mathcal{H})$, допускающих представление вида

$$
X=X_{l} F, \quad X=F X_{r},
$$

где $X_{l}, X_{r} \in \mathfrak{S}_{2}(\mathcal{H})$. Положим $\|X\|_{\mathcal{M}_{B}}=\max \left\{\left\|X_{l}\right\|_{2},\left\|X_{r}\right\|_{2}\right\}$. Очевидно, что $\|X\|_{2} \leqslant\|X\|_{\mathcal{M}_{B}}$, $X \in \mathcal{M}_{B}$.

Свойство Ker $F=0$ (см. свойство (5) последовательности $\alpha_{n}(X)$ ) позволяет заключить, что введенное множество операторов $\mathcal{M}_{B}$ является банаховым пространством.

Очевидно, что для любого оператора $X$ из $\mathfrak{S}_{2}(\mathcal{H})$ имеет место представление

$$
X=\left(\sum_{n \in \mathbb{Z}} \frac{1}{\alpha_{n}(X)} X P_{n}\right) F_{X}=F_{X}\left(\sum_{n \in \mathbb{Z}} \frac{1}{\alpha_{n}(X)} P_{n} X\right) .
$$

Поэтому возмущение $B$ принадлежит пространству $\mathcal{M}_{B}$.

Отметим, что последовательность $\alpha: \mathbb{Z} \rightarrow \mathbb{R}_{+}$характеризует скорость убывания матричных элементов оператора $X \in \mathfrak{S}_{2}(\mathcal{H})$ по строкам и столбцам.

Поскольку $\mathcal{M}_{B} \subset \mathfrak{S}_{2}(\mathcal{H})$, то трансформаторы $J_{k}$ и $\Gamma_{k}, k \geqslant 0$, задаваемые формулами (4.1), (4.3), (4.4), определены и для операторов из $\mathcal{M}_{B}$. Более того, подпространство $\mathcal{M}_{B}$ инвариантно относительно $J_{k}$ и $\Gamma_{k}, k \geqslant 0$, и

$$
\begin{aligned}
& J_{k}\left(X_{l} F\right)=\left(J_{k} X_{l}\right) F, \quad J_{k}\left(F X_{r}\right)=F\left(J_{k} X_{r}\right), \\
& \Gamma_{k}\left(X_{l} F\right)=\left(\Gamma_{k} X_{l}\right) F, \quad \Gamma_{k}\left(F X_{r}\right)=F\left(\Gamma_{k} X_{r}\right),
\end{aligned}
$$

где $X_{r}, X_{l} \in \mathfrak{S}_{2}(\mathcal{H})$.

Для оценки норм $\left\|\Gamma_{k}(X F)\right\|_{2}$ и $\left\|\Gamma_{k}(F X)\right\|_{2}, X \in \mathfrak{S}_{2}(\mathcal{H})$, рассмотрим две последовательности $\left(\alpha_{n}^{\prime}\right), n \in \mathbb{N}$, и $\left(\widetilde{\alpha}_{n}^{\prime}\right), n \in \mathbb{N}$, определенные формулами

$$
\begin{gathered}
\alpha_{n+1}^{\prime}=\max \left\{\lambda_{l} d_{j l}^{-1}, l, j \in \mathbb{Z},|l| \leqslant n,|j|>n\right\}, \\
\widetilde{\alpha}_{n}^{\prime}=\frac{\omega}{2 \pi} \alpha_{n}+\alpha_{n}^{\prime}, \quad n \in \mathbb{N},
\end{gathered}
$$

где $d_{i j}=\operatorname{dist}\left(\sigma_{i}, \sigma_{j}\right), i, j \in \mathbb{Z}$. Последовательности $\left(\alpha_{n}^{\prime}\right)$ и $\left(\widetilde{\alpha}_{n}^{\prime}\right)$ принадлежат пространству $c_{0}$ сходящихся к нулю последовательностей, т.е.

$$
\lim _{n \rightarrow \infty} \alpha_{n+1}^{\prime}=\lim _{n \rightarrow \infty} \widetilde{\alpha}_{n}^{\prime}=0 .
$$

Следующая лемма является аналогом леммы 3 из [9].

Лемма 5.1. Для всех $k \in \mathbb{Z}_{+} u X \in \mathfrak{S}_{2}(\mathcal{H})$ имеют место оценки

$$
\max \left\{\left\|\Gamma_{k}(X F)\right\|_{2},\left\|\Gamma_{k}(F X)\right\|_{2}\right\} \leqslant \widetilde{\alpha}_{k+1}\|X\|_{2} .
$$

Доказательство. Пусть $P^{(k)}=I-P_{(k)}, k \in \mathbb{Z}_{+}$, - дополнительный к $P_{(k)}$ проектор. Тогда

$$
\left\|F P^{(k)}\right\|=\left\|\sum_{n \in \mathbb{Z}} \alpha_{n} P_{n} P^{(k)}\right\| \leqslant \alpha_{k+1} .
$$

Из определения трансформатора $\Gamma_{k}$ следует

$$
\Gamma_{k}(X F)=\Gamma_{k}\left(X F P^{(k)}\right)+\Gamma_{k}\left(P^{(k)} X F P_{(k)}\right) .
$$

Выпишем матрицы операторов $\Gamma_{k}\left(X F P^{(k)}\right), \Gamma_{k}\left(P^{(k)} X F P_{(k)}\right)$ :

$$
\begin{aligned}
\left(\Gamma_{k}\left(X F P^{(k)}\right)\right)_{j l} & = \begin{cases}\alpha_{l} X_{j l} /\left(\lambda_{j}-\lambda_{l}\right), & |l|>k, l \neq j, \\
0 & \text { в остальных случаях, }\end{cases} \\
\left(\Gamma_{k}\left(P^{(k)} X F P_{(k)}\right)\right)_{j l} & = \begin{cases}\alpha_{l} X_{j l} /\left(\lambda_{j}-\lambda_{l}\right), & |l| \leqslant m,|j|>m, \\
0 & \text { в остальных случаях. }\end{cases}
\end{aligned}
$$


Следовательно,

$$
\left\|\Gamma_{k}\left(X F P^{(k)}\right)\right\|_{2} \leqslant \frac{\omega}{2 \pi} \alpha_{k+1}\|X\|_{2}^{2}, \quad\left\|\Gamma_{k}\left(P^{(k)} X F P_{(k)}\right)\right\|_{2} \leqslant \alpha_{k+1}^{\prime}\|X\|_{2}^{2} .
$$

Таким образом, для $\left\|\Gamma_{k} X F\right\|_{2}$ оценка установлена. Аналогично и той же величиной оценивается $\left\|\Gamma_{k}(F X)\right\|_{2}$. Лемма 5.1 доказана.

Теорема 5.1. Тройка $\left(\mathcal{M}_{B}, J_{k}, \Gamma_{k}\right)$ является допустимой тройкой для невозмущенного оператора $L_{0}$ для любого $k \in \mathbb{Z}_{+}$, а постолнная $\gamma=\gamma_{k}$ из определения 2.3 допускает оченку

$$
\gamma_{k} \leqslant \widetilde{\alpha}_{k+1}^{\prime}, \quad k \in \mathbb{Z}
$$

Доказательство. Ранее было установлено, что $\mathcal{M}_{B}$ является банаховым пространством. Из вложения $\mathcal{M}_{B} \subset \mathfrak{S}_{2}(\mathcal{H})$ следует, что $\mathcal{M}_{B} \subset \mathfrak{L}_{L_{0}}(\mathcal{H})$.

Выполнение свойств $(2),(3)$ и (5) следует из построения трансформаторов $J_{k}, \Gamma_{k} \in \operatorname{End}\left(\mathfrak{S}_{2}(\mathcal{H})\right)$, $k \geqslant 0$.

Перейдем к свойству (4). Пусть $X=X_{l} F \in \mathcal{M}_{B}, Y=Y_{l} F \in \mathcal{M}_{B}, X_{l}, Y_{l} \in \mathfrak{S}_{2}(\mathcal{H})$. Тогда $X \Gamma_{k} Y=X_{l} F \Gamma_{k} Y_{l} F=Z_{l} F$, где $Z_{l}=X_{l} F \Gamma_{k} Y_{l}$. Из леммы 5.1 следует, что

$$
\left\|Z_{l}\right\|_{2} \leqslant \widetilde{\alpha}_{k+1}^{\prime}\left\|X_{l}\right\|_{2}\left\|Y_{l}\right\|_{2} \leqslant \widetilde{\alpha}_{k+1}^{\prime}\|X\|_{\mathcal{M}_{B}}\|Y\|_{\mathcal{M}_{B}} .
$$

Аналогично устанавливаются остальные оценки в свойстве (4). Теорема 5.1 доказана.

6. Основные результаты. Отметим, что в данном случае операторы $L_{0}$ и $B$ не являются самосопряженными, поэтому мы будем использовать оператор преобразования $U=I+\Gamma X$ и уравнение (2.6).

Из теорем 4.1 и 2.2 вытекает следующее утверждение.

Теорема 6.1. Пусть для оператора В выполнено условие

$$
\|B\|_{2}<\frac{\pi}{2 \omega}
$$

Тогда оператор $L_{0}-B$ подобен оператору $L_{0}-J_{k} X_{*}=L_{0}-V, X_{*}, V \in \mathfrak{S}_{2}(\mathcal{H})$, имеющему диагональную операторную матрицу. Имеет место равенство

$$
\left(L_{0}-B\right)\left(I+\Gamma X_{*}\right)=\left(I+\Gamma X_{*}\right)\left(L_{0}-V\right),
$$

где оператор $X_{*} \in \mathfrak{S}_{2}(\mathcal{H})$ - решение нелинейного операторного уравнения (2.6).

Отметим, что условие (6.1) довольно жесткое и не всегда выполняется. Поэтому обычно бывает удобно рассмотреть допустимую тройку $\left(\mathcal{M}_{B}, J_{k}, \Gamma_{k}\right)$ из раздела 5.

Теорема 6.2. Пусть иелое число $k \geqslant 0$ таково, что выполняется неравенство

$$
4 \widetilde{\alpha}_{k+1}^{\prime}\|B\|_{\mathcal{M}_{B}}<1 .
$$

Тогда оператор $L_{0}-B$ подобен блочно-диагональному оператору

$$
L_{0}-J_{k} X_{*}=L_{0}-P_{(k)} X_{*} P_{(k)}-\sum_{\substack{|i|>k, i \in \mathbb{Z}}} P_{i} X_{*} P_{i}=L_{0}-V,
$$

где $X_{*} \in \mathcal{M}_{B}$ - решение нелинейного уравнения (2.6). Оператор преобразования оператора $L_{0}-B$ в onepamop $L_{0}-V$ ecms onepamop $I+\Gamma X_{*}, \Gamma X_{*} \in \mathcal{M}_{B} \subset \mathfrak{S}_{2}(\mathcal{H})$.

Далее через $l_{p}=l_{p}(\mathbb{Z}), 1 \leqslant p<\infty$, обозначим банахово пространство двусторонних числовых последовательностей $x: \mathbb{Z} \rightarrow \mathbb{C}$, суммируемых со степенью $p$, с нормой

$$
\|x\|_{p}=\left(\sum_{n \in \mathbb{Z}}|x(n)|^{p}\right)^{1 / p}, \quad x \in l_{p} .
$$

Из теорем 6.1, 6.2 и леммы 2.1 получаются следующие утверждения, касающиеся асимптотики спектра возмущенного оператора $L$. 
Теорема 6.3. Пусть выполнено условие (6.1). Тогда спектр $\sigma(L)$ оператора $L$ представим в виде объединения простых изолированных собственных значений $\widetilde{\lambda}_{n}, n \in \mathbb{Z}$ :

$$
\sigma(L)=\bigcup_{n \in \mathbb{Z}}\left\{\tilde{\lambda}_{n}\right\}
$$

где

$$
\widetilde{\lambda}_{n}=i \frac{2 \pi n}{\omega}-\left(B e_{n}, e_{n}\right)+\beta_{n}
$$

u последовательность $\left(\beta_{n}\right)$ принадлежит $l_{1}$.

Доказательство. Первое утверждение теоремы 6.3 следует из теоремы 6.1 и леммы 2.1. Для доказательства формулы (6.2) применим к обеим частям уравнения (2.6) проекторы $P_{n}$ слева и справа и, учитывая свойство (5) определения 2.3, получим

$$
P_{n} X_{*} P_{n}=P_{n}\left(B \Gamma_{m} X_{*}\right) P_{n}+P_{n} B P_{n} .
$$

При этом оператор $B \Gamma X_{*} \in \mathfrak{S}_{1}(\mathcal{H})$ как произведение двух операторов Гильберта-Шмидта. Напомним также, что оператор $L_{0}-J X_{*}$ диагональный и образ проектора $P_{n}, n \in \mathbb{Z}$, одномерный. Следовательно,

$$
\sigma\left(L_{0}-J X_{*}\right)=\bigcup_{n \in \mathbb{Z}}\left\{\lambda_{n}-x_{* n n}\right\},
$$

где $x_{* n n}=\left(X_{*} e_{n}, e_{n}\right), n \in \mathbb{Z}$. Из (6.3) вытекает, что $x_{* n n}=\left(B e_{n}, e_{n}\right)+\beta_{n}, n \in \mathbb{Z}$, где последовательность $\beta_{n}$ принадлежит $l_{1}$. Теорема 6.3 доказана.

Теорема 6.4. В условиях теоремы 6.2 спектр $\sigma(L)$ оператора $L$ представим в виде

$$
\sigma(L)=\widetilde{\sigma}_{(n)} \cup\left\{\widetilde{\lambda}_{k},|k|>n\right\}
$$

где множество $\widetilde{\sigma}_{(n)}, n \in \mathbb{Z}_{+}$, содержит не более чем $2 n+1$ собственных значений, собственные значения $\widetilde{\lambda}_{k},|k|>n$, простье изолированнье,

$$
\widetilde{\lambda}_{k}=i \frac{2 \pi k}{\omega}-\left(B e_{k}, e_{k}\right)+\beta_{k}^{\prime}
$$

u последовательность $\beta_{k}^{\prime}$ принадлежит $l_{1}$.

Теорема 6.5. Собственные векторы $\widetilde{e}_{i}, i \in \mathbb{Z}$, оператора $L$ образуют в пространстве $\mathcal{H}$ базис Бари (в частности, базис Рисса) и р-базис для $p \geqslant 1$. Имеют место следующие формуль:

$$
\widetilde{e}_{i}=e_{i}+\Gamma_{k} X_{*} e_{i}, \quad k \in \mathbb{Z}_{+},
$$

где $X_{*}$ - решение нелинейного операторного уравнения (2.6),

$$
\begin{array}{ll}
\left\|\widetilde{e}_{i}-e_{i}\right\|=\delta_{i}, & |i|>k, \\
\left\|\widetilde{e}_{i}-e_{i}-\Gamma_{k} B e_{i}\right\|=\left\|\widetilde{e}_{i}-e_{i}+\frac{i \omega}{2 \pi} \sum_{j \neq i} \frac{B e_{i}}{j-i} e_{j}\right\|=\delta_{i}^{\prime}, & |i|>k .
\end{array}
$$

где последовательность $\delta_{i},|i|>k$, принадлежит $l_{2}$, а последовательность $\delta_{i}^{\prime},|i|>k$, принадлежит $l_{1}$.

Утверждение теоремы 6.5 следует из леммы 2.1 и принадлежности оператора $Г X_{*}$ идеалу $\mathfrak{S}_{2}(\mathcal{H})$, а оператора $\Gamma\left(X_{*}-B\right)$ идеалу $\mathfrak{S}_{1}(\mathcal{H})$.

Перейдем к оценке спектральных проекторов. Обозначим через $\Omega$ произвольное множество из $\mathbb{Z} \backslash\{-k, \ldots, 0,1, \ldots, k\}$. Для множества $\Delta=\Delta(\Omega)=\left\{\lambda_{n}, n \in \Omega\right\}$ проектор $P_{\Delta}=P\left(\Delta, L_{0}\right)$ определим равенством

$$
P_{\Delta} x=\sum_{n \in \Omega} P_{n} x, \quad x \in \mathcal{H}
$$

Аналогично,

$$
\widetilde{P}_{i}=P\left(\left\{\widetilde{\lambda}_{i}\right\}, L\right), \quad i \in \mathbb{Z}, \quad \widetilde{P}_{(k)}=P\left(\widetilde{\sigma}_{k}, L\right), \quad \widetilde{P}_{\Delta} x=\sum_{i \in \mathbb{Z}} \widetilde{P}_{i} x, \quad x \in \mathcal{H} .
$$


Лемма 6.1. Пусть имеют место условия теоремы 6.1. Тогда для спектральных проекторов $\widetilde{P}_{i},|i|>k, \widetilde{P}_{(k)}$ и $\widetilde{P}_{\Delta}$ справедливы следуюшие формуль:

$$
\begin{aligned}
& \widetilde{P}_{i}=P_{i} U^{-1}+\Gamma X_{*} P_{i} U^{-1}, \quad \widetilde{P}_{i}-P_{i}=\left(\Gamma_{k} X_{*} P_{i}-P_{i} \Gamma_{k} X_{*}\right) U^{-1}, \\
& \widetilde{P}_{(k)}=P_{(k)} U^{-1}+\Gamma_{k} X_{*} P_{(k)} U^{-1}, \quad \widetilde{P}_{(k)}-P_{(k)}=\left(\Gamma_{k} X_{*} P_{(k)}-P_{(k)} \Gamma_{k} X_{*}\right) U^{-1}, \\
& \widetilde{P}_{\Delta}=P_{\Delta} U^{-1}+\Gamma_{k} X_{*} P_{\Delta} U^{-1}, \quad \widetilde{P}_{\Delta}-P_{\Delta}=\left(\Gamma_{k} X_{*} P_{\Delta}-P_{\Delta} \Gamma_{k} X_{*}\right) U^{-1} .
\end{aligned}
$$

Все приведенные в лемме 6.1 формулы следуют из представления проекторов подобных операторов в виде $\widetilde{P}_{i}=U P_{i} U^{-1},|i|>k$; аналогично для проекторов $\widetilde{P}_{(k)}$ и $\widetilde{P}_{\Delta}$.

Непосредственно из леммы 6.1 вытекает следующее утверждение.

Теорема 6.6. В условиях теорем 6.1 и 6.2 для спектралъных проекторов $\widetilde{P}_{j}, j>k, u \widetilde{P}_{\Delta}$ имегт место оценки

$$
\left\|\widetilde{P}_{j}-P_{j}\right\|_{2} \leqslant M \nu(j),
$$

где $\nu: \mathbb{Z} \rightarrow \mathbb{R}$ - последовательность из $l_{2}$ и $M$-некоторая константа.

Доказательство. Так как

$$
\left\|\widetilde{P}_{j}-P_{j}\right\|_{2} \leqslant\left\|U^{-1}\right\|\left(\left\|\Gamma_{k} X_{*} P_{j}\right\|_{2}+\left\|P_{j} \Gamma_{k} X_{*}\right\|_{2}\right) \leqslant M \max \left\{\left\|X_{*} P_{j}\right\|_{2},\left\|P_{j} X_{*}\right\|_{2}\right\},
$$

то оценка установлена. При этом последовательности $\left\|X_{*} P_{j}\right\|_{2},|j|>k$, а также $\left\|P_{j} X_{*}\right\|_{2},|j|>k$, суммируемы с квадратом, так как $X_{*} \in \mathfrak{S}_{2}(\mathcal{H})$.

В заключение этого раздела отметим, что аналогично $[11,25,27]$ можно выписать группу операторов, генератором которой является исследуемый оператор $L$.

7. Пример. Пусть $\omega=1$ и

$$
(B y)(t)=C \int_{0}^{1} t(t+s) y(s) d s,
$$

где $C>0$ - некоторая константа. Непосредственный подсчет показывает, что $\|B\|_{2}=C \sqrt{101 / 180}$ и возмущение $B$ имеет числовую матрицу $B \sim\left(b_{n m}\right), n, m \in \mathbb{Z}$, состоящую из элементов

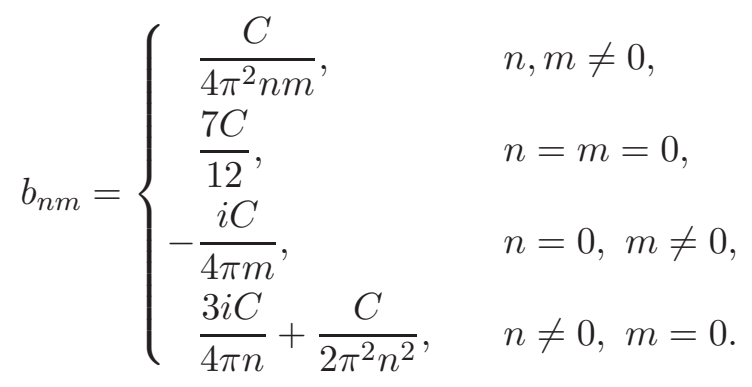

Возмущение $B$ не является самосопряженным оператором, и сопряженный оператор $B^{*}$ задается формулой

$$
\left(B^{*} y\right)(t)=\int_{0}^{1} s(t+s) y(s) d s .
$$

Из теорем 6.1-6.3 вытекает следующее утверждение.

Теорема 7.1. Пусть число $C$ таково, что выполняется условие

$$
\frac{2 C}{\pi} \sqrt{\frac{101}{180}}<1
$$

Тогда оператор $L$ подобен оператору $L_{0}-V$, где $V \in \mathfrak{S}_{2}(\mathcal{H})$, оператор $V=J X_{*}$ имеет диагональную матричу и $X_{*}$ есть решение уравнения (2.6). Имеют место следуюшие оценки собственных значений оператора $L$ :

$$
\widetilde{\lambda}_{0}=-\frac{7 C}{12}+\beta_{0}, \quad \widetilde{\lambda}_{n}=i 2 \pi n-\frac{C}{4 \pi^{2} n^{2}}+\beta_{n}
$$


где последовательность $\left(\beta_{n}, n \in \mathbb{Z}\right)$ принадлежит $l_{1}$.

Если же число $C$ не удовлетворяет условию (7.1), то оператор $L$ подобен блочно-диагональному оператору $L_{0}-\widetilde{V}$, где $\widetilde{V} \in \mathfrak{S}_{2}(\mathcal{H}), \widetilde{V}=J_{k} X_{*}$ для некоторого $k \in \mathbb{Z}_{+}$u оператор $X_{*} \in \mathfrak{S}_{2}(\mathcal{H})$ есть решение нелинейного уравнения (2.6) с операторами $J_{k}, \Gamma_{k} \in \operatorname{End}\left(\mathfrak{S}_{2}(\mathcal{H})\right.$. Имеют место оценки

$$
\widetilde{\lambda}_{n}=i 2 \pi n-\frac{C}{4 \pi^{2} n^{2}}+\beta_{n}, \quad|n|>k,
$$

$u$ последовательность $\left(\beta_{n},|n|>k\right)$ принадлежит $l_{1}$.

Отметим также, что в работах $[11-13,24,25,27]$, использующих последовательность $\alpha_{n}$ из раздела 5 , обычно не приводится конкретный вид этой последовательности. Ниже мы выпишем для рассматриваемого примера последовательность $\alpha_{n}(B), n \in \mathbb{Z}$. Сначала необходимо вычислить нормы операторов $\left\|B P_{n}\right\|_{2}$ и $\left\|P_{n} B\right\|_{2}, n \in \mathbb{Z}$. Отметим, что в силу одномерности образов проекторов $P_{n}, n \in \mathbb{Z}$, имеем $\left\|B P_{n}\right\|_{2}=\left\|B e_{n}\right\|$ и $\left\|P_{n} B\right\|_{2}=\left\|B^{*} e_{n}\right\|$. Непосредственное вычисление показывает, что

$$
\begin{array}{ll}
\left(B e_{n}\right)(t)=\frac{t}{i 2 \pi n}, & \left(B e_{0}\right)(t)=\frac{1}{2}\left(2 t^{2}+t\right), \\
\left(B^{*} e_{n}\right)(t)=\frac{t+1}{i 2 \pi n}-\frac{1}{2 \pi^{2} n^{2}}, & \left(B^{*} e_{0}\right)(t)=\frac{t}{2}+\frac{1}{3}, \\
\left\|B e_{n}\right\|^{2}=\frac{1}{12 \pi^{2} n^{2}}, & \left\|B^{*} e_{n}\right\|=\frac{1}{4 \pi^{4} n^{4}}+\frac{7}{12 \pi^{2} n^{2}}, \\
\left\|B e_{0}\right\|^{2}=\frac{8}{15}, & \left\|B^{*} e_{0}\right\|=\frac{13}{36}, \\
\sum_{|j|>n}\left\|B P_{j}\right\|^{2}=\sum_{|j|>n} \frac{1}{12 \pi^{2} j^{2}} \leqslant \frac{1}{36 \pi^{2}(n-1)}, \quad n>1, \\
\sum_{|j|>n}\left\|P_{j} B\right\|^{2}=\sum_{|j|>n}\left(\frac{1}{4 \pi^{4} j^{4}}+\frac{7}{12 \pi^{2} j^{2}}\right) \leqslant \frac{1}{20 \pi^{4}(n-1)^{5}}+\frac{7}{36 \pi^{2}(n-1)^{3}} .
\end{array}
$$

Тогда $\alpha_{n}(B)$ при $|n|>1$ допускает оценку

$$
\alpha_{n}(B) \leqslant\left(\frac{180}{101}\left(\frac{1}{20 \pi^{4}(n-1)^{5}}+\frac{7}{36 \pi^{2}(n-1)^{3}}\right)\right)^{1 / 4}
$$

Последовательность $\alpha_{n}(B)$ характеризует скорость убывания матричных элементов возмущения $B$. Так как решение нелинейного операторного уравнения $(2.6)$ также принадлежит $\mathcal{M}_{B}$, то неизвестный оператор $X_{*}$ наследует эту скорость.

\section{СПИСОК ЛИТЕРАТУРЫ}

1. Баскаков А. Г. Замена Крылова-Боголюбова в теории возмущений линейных операторов/ Препринт ИПМ им. М. В. Келдыша. - М., 1980.

2. Баскаков А. Г. Методы абстрактного гармонического анализа в теории возмущений линейных операторов// Сиб. мат. ж. - 1983. - 24, № 1. - С. 21-39.

3. Баскаков А. Г. Замена Крылова-Боголюбова в теории возмущений линейных операторов// Укр. мат. ж. - 1984. - 36. - С. 601-611.

4. Баскаков А. Г. Метод усреднения в теории возмущений линейных дифференциальных операторов// Диффер. уравн. - 1985. - 21, № 4. - С. 555-562.

5. Баскаков А. Г. Теорема о расщеплении оператора и некоторые смежные вопросы аналитиеской теории возмущений// Изв. АН СССР. Сер. мат. - 1986. - 50, № 3. - С. 435-457.

6. Баскаков А. Г. Гармонический анализ линейных операторов. - Воронеж: ВГУ, 1987.

7. Баскаков А. Г. Спектральный анализ возмущенных неквазианалитических и спекральных операторов// Изв. РАН. Сер. мат. - 1994. - 58, № 4. - С. 3-32.

8. Баскаков А. Г. Об абстрактном аналоге преобразования Крылова-Боголюбова в теории возмущений линейных операторов// Функц. анал. прилож. - 1999. - 32, № 2. - С. 76-80.

9. Баскаков А. Г., Дербушев А. В., Щербаков А. О. Метод подобных операторов в спектральном анализе оператора Дирака с негладким потенциалом// Изв. РАН. Сер. мат. - 2011. - 75, № 3. - С. 3-28. 
10. Баскаков А. Г., Поляков Д. М. Метод подобных операторов в спектральном анализе оператора Хилла с негладким потенциалом// Мат. сб. - 2017. - 208, № 1. - С. 3-47.

11. Баскаков А. Г., Ускова Н. Б. Метод Фурье для дифференциальных уравнений первого порядка и группы операторов// Уфим. мат. ж. - 2018. - 10, № 3. - С. 11-34.

12. Баскаков А. Г., Ускова Н. Б. Обобщенный метод Фурье для системы дифференциальных уравнений первого порядка и группы операторов// Диффер. уравн. - 2018. - 54, № 2. - С. 276-280.

13. Баскаков А. Г., Ускова Н. Б. Спектральный анализ дифференциальных операторов с инволюцией и группы операторов// Диффер. уравн. - 2018. - 54, № 9. - С. 1287-1291.

14. Бурлуикая М. Ш. О смешанной задаче для уравнения с частными производными первого порядка с инволюцией и с периодическими краевыми условиями // Ж. выч. мат. мат. физ. - 2014. - 54, № 1 . C. $3-12$.

15. Бурлуцкая М. Ш., Курдюмов В. П., Луконина А. С., Хромов А. П. Функционально-дифференциальный оператор с инволюцией// Докл. РАН. - 2007. - 414, № 3. - С. 443-446.

16. Бурлуцкая М. Ш., Хромов А. П. Классическое решение для смешанной задачи с инволюцией// Докл. PAH. - 2010. - 435, № 2. - C. 151-154.

17. Бурлуцкая M. Ш., Хромов А. П. Метод Фурье в смешанной задачи для уравнения с частными производными первого порядка с инволюцией// Ж. выч. мат. мат. физ. - 2011. - 51, № 12. - С. $2233-2245$.

18. Бурлучкая М. Ш., Хромов А. П. Смешанные задачи для гиперболических уравнений первого порядка// Докл. РАН. - 2011. - 441, № 2. - С. 156-159.

19. Бурлуцкая М. Ш., Хромов А. П. Теорема Штейнгауза о равносходимости для функциональнодифференциальных операторов// Мат. заметки. - 2011. - 90, № 1. - С. 22-33.

20. Бурлуцкая М. Ш., Хромов А. П. Функционально-дифференциальные операторы с инволюцией и операторы Дирака с периодическими краевыми условиями// Докл. РАН. - 2014. - 454, № 1. - С. 15-17.

21. Гохберг И. Ц., Крейн М. Г. Введение в теорию несамосопряженных операторов в гильбертовом пространстве. - М.: Наука, 1965.

22. Рудин У. Функциональный анализ. - М.: Мир, 1975.

23. Ускова Н. Б. К одному результату Р. Тернера// Мат. заметки. - 2004. - 76, № 6. - С. $905-917$.

24. Baskakov A. G., Krishtal I. A., Romanova E. Yu. Spectral analysis of a differential operator with an involution// J. Evolut. Eqs. - 2017. - 17. - P. 669-684.

25. Baskakov A. G., Krishtal I. A., Uskova N. B. Linear differential operator with an involution as a generator of an operator group// J. Oper. Matr. — 2018. — 12, № 3. — P. 723-756.

26. Baskakov A. G., Krishtal I. A., Uskova N. B. General Dirac operators as generators of operator groups/ arXiv: 1806.10831 [math.SP].

27. Baskakov A. G., Krishtal I. A., Uskova N. B. Similarity techniques in the spectral analysis of perturbed operator matrices/ arXiv: 1812.10331 [math.SP].

28. Friedrichs K. O. Lectures on Advanced Ordinary Differential Equations. - New York: Gordon and Breach, 1965.

29. Turner R. F. L. Perturbation of compact spectral operators// Commun. Pure. Appl. Math. - 1965. 18. - P. 519-541.

Баскаков Анатолий Григорьевич

Воронежский государственный университет;

Северо-Осетинский государственный университет им. К. Л. Хетагурова, Владикавказ

E-mail: anatbaskakov@yandex.ru

Криштал Илья Аркадьевич

Университет Северного Иллинойса, Де-Калб, Иллинойс, США

E-mail: ikrishtal@niu.edu

Ускова Наталья Борисовна

Воронежский государственный технический университет

E-mail: nat-uskova@mail.ru 